\title{
Patient-centered medicine and patient-oriented research: improving health outcomes for individual patients
}

\author{
José A Sacristán
}

\begin{abstract}
Background: Patient-centered medicine is developing alongside the concepts of personalized medicine and tailored therapeutics. The main objective of patient-centered medicine is to improve health outcomes of individual patients in everyday clinical practice, taking into account the patient's objectives, preferences, values as well as the available economic resources.

Discussion: Patient-centered medicine implies a paradigm shift in the relationship between doctors and patients, but also requires the development of patient-oriented research. Patient-oriented research should not be based on the evaluation of medical interventions in the average patient, but on the identification of the best intervention for every individual patient, the study of heterogeneity and the assignment of greater value to observations and exceptions. The development of information-based technologies can help to close the gap between clinical research and clinical practice, a fundamental step for any advance in this field.

Summary: Evidence-based medicine and patient centered medicine are not contradictory but complementary movements. It is not possible to practice patient-centered medicine that is not based on evidence, nor is it possible to practice evidence-based medicine at a distance from the individual patient.
\end{abstract}

Keywords: Patient-centered care, Research, Preferences, Patient-reported outcomes, Heterogeneity, Clinical trials

\section{Background}

Patients are playing an ever-greater role in the healthcare systems of developed countries. The healthcare systems in these countries are evolving from a doctor-patient relationship based on paternalism and the authority of the doctor to a model based on the ethical principle of patients' autonomy, where patients are transforming into customers, have more and better information, and want to take a more active role in making decisions that affect them [1]. These changes are reflected by the principal of "nothing about me without me" and the development of patient-centered medicine (PCM) [2].

Patients have always been the focus of medical care, which means the concept of PCM seems to be redundant and provocative. However, emphasizing the existence of a healthcare process that is focused on the patient implies

Correspondence: sacristan_jose@lilly.com

Clinical Research Department, Lilly Spain, Avda. de la Industria, 30, Alcobendas, Madrid 28108, Spain explicit recognition that other ways of practicing medicine exist. In fact, in the last few decades, healthcare has been characterized by a provider-centered model [2] with an emphasis on the evaluation and treatment of diseases rather than the evaluation and treatment of patients. These and other characteristics of PCM, compared with those of traditional clinical medicine, are summarized in Table 1.

PCM is developing alongside the concepts of personalized medicine and tailored therapeutics [3]. Advances in pharmacogenomics and personalized medicine, the objective of which is to provide tailored therapeutic approaches, can be summed up with the catch-phrase "one size does not fit all". However, the concept of PCM is broader than personalized medicine, as far as the term is usually understood. Personalized medicine reaches it full significance as the opposite of "depersonalized medicine" or "illnessoriented care", the aim of which is to treat the illness, not the patients with the illness [4]. The practice of PCM requires the dedication of greater effort to improve our 
Table 1 Characteristics of the traditional medical model and patient-centered medicine

\begin{tabular}{ll}
\hline Traditional medical model & Patient-centered medicine \\
\hline Provider-centered model & Patient-centered model \\
\hline Founded on the principles of beneficence and authoritarianism & Founded on the principle of autonomy \\
\hline Disease-oriented care & Patient-oriented care \\
\hline Focuses on outcomes of importance for physicians and regulators & Focuses on outcomes of importance for patients \\
\hline The patient's perspective is usually ignored & $\begin{array}{l}\text { The patient's preferences, objectives and values are taken into } \\
\text { account during decision making and delivery of healthcare }\end{array}$ \\
\hline Compliance with the physician's decisions & The patient and physician share decision making \\
\hline Improve outcomes for the average patient & Improve outcomes for the individual patient \\
\hline Population-oriented research & Patient-oriented research \\
\hline
\end{tabular}

knowledge of the psychological, social and cultural aspects that may affect disease prognosis in individual patients.

PCM can be defined as the medical practice aimed at improving the health outcomes of individual patients in everyday clinical practice, taking into account their preferences, objectives and values, as well as the available economic resources. PCM requires a change in the relationship between the doctor and the patient, and also has implications on medical research, legislation, education, and medical ethics. This work discusses the objectives and characteristics of PCM, and the implications of this model for medical research and clinical practice.

\section{Discussion}

\section{Objectives of PCM}

If the objective of PCM is to improve the health outcomes for individual patients, then we need to define the outcomes that should be measured, and the significance of improving these outcomes. In research to date, the outcome measures are usually those that are most important to the doctors and regulators. Through PCM, the focus should shift to outcomes that are important to the patients themselves [5]. For example, in the context of diabetes, only $18 \%$ of the randomized clinical trials (RCTs) evaluated included outcomes that are important to patients, such as amputations, stroke, loss of vision, and other outcomes that affect quality of life [6]. Similarly, in cardiovascular disease, only $16 \%$ of studies included at least one instrument used to measure patient-reported outcomes (PRO) [7].

Clearly, it is necessary to measure final outcomes, not just processes such as compliance to good practice guidelines achieved by the doctors [8]. Although "hard" outcomes such as mortality or serious complications should be measured, "soft" outcomes such as symptoms, physical functional status, social and role functions, or quality of life-"goal-oriented patient care outcomes"-better reflect the patients' perspective [9]. Patient-centered care means attending to the physiological and social aspects of an illness that cannot be reduced to a single objective measure.
Sometimes, evidence on patient preferences might only be captured with qualitative research, not from questionnaires or blood parameters [10].

The regulatory authorities are now promoting the use of PRO, and both the EMA and the FDA have published guidelines to include these measures in RCTs, so that the labels of the medicines can include this information $[11,12]$. Many scales designed to measure PRO were published in recent years, although these publications were not always accompanied by appropriate validation. For example, in the areas of diabetes and schizophrenia, only $44 \%$ [13] and 35\% [14], respectively, of the variables have been adequately validated.

Referring to what signifies a relevant improvement in outcomes, we need to remember that " $a$ difference, to be a difference, must make a difference." A statistically significant difference does not necessarily represent a clinically relevant difference. Clinical relevance is related to the concept of clinical usefulness, "a multifactorial parameter that describes the relevance and end usefulness of an intervention in patient care" [15]. From the point of view of PCM, clinical usefulness should consider the improvements in outcomes that are important to the patients (i.e., survival, quality of life and functional ability), and establish the threshold and the lowest level of change considered relevant by the patients $[16,17]$. Clinical usefulness should be conceptualized to include the dimension of "personal utility" for individual patients [15].

An essential element of PCM is the active participation of patients in the decision-making process [18]. The concept of shared decision-making necessitates a profound change in the traditional model of the doctorpatient relationship [19]. This new model is based on greater information exchange. In particular, the doctor must provide the patient with full and comprehensible information on the disease, prognosis, and the potential benefits and risks of the different therapeutic options available. In turn, the patient must provide the doctor with preferences and attitudes towards the disease and the risks involved [20]. A well-informed patient is 
essential to ensure the decision-making can be based on the patient's preferences. For the doctor, the fundamental change is not in the individualization of treatments, but in the individualization of therapeutic decisions, where the patient's preferences play a fundamental role.

Patients and doctors need to be educated in this new paradigm. The role of the healthcare professional should change "from experts who care for patients to enablers who support patients to make decisions" [20]. The doctor should be able to adapt the information available to suit the requirements of individual patients, so that the patient, after giving informed consent, can establish personal trade-offs for the available treatments [21]. Factors such as level of adherence to medication, degree of tolerance to an adverse effect, past experience, and health objectives can have a decisive influence on the patient's preferences.

In many occasions, there is one clearly superior path, in which case the patient's preferences may play little or no role. Generally, patients evaluate prevention and increased life expectancy positively [21]. However, in many medical decisions, there is more than one reasonable option [19]. For example, a patient may prefer a more economical treatment even if it is less effective, a more conservative surgery because of greater emphasis on quality of life than on life expectancy, or may refuse chemotherapy because the adverse effects are considered unlikely to compensate for the increased survival time [21]. Fortunately, obtaining information about a patient's preferences does not require the use of complex scales or sophisticated statistical tests. A good anamnesis, and asking the patient what he prefers, is an excellent strategy for personalized research. If personalized medicine is determined by the patient's preferences [22], it seems reasonable that this information should be included in their clinical histories [1].

For the patients, decision aids have been developed that could help them in this process [23], but the patients would need to fundamentally change their attitudes and have a greater level of literacy to fully benefit from these aids. Patients must clearly describe their expectations and choices, and accept the uncertainty that surrounds each medical decision. The patients must also understand that their responsibility is to not just follow their doctors' orders but also take joint responsibility for the decisions and therapeutic strategies agreed with their doctors, and accept the results of these decisions. Providing patients with greater empowerment over medical decisions involves a switch from the current situation of unconscious certainty to a situation of conscious uncertainty.

PCM can only be achieved through the development of patient-oriented research, the results of which could be translated into clinical decisions that increase the effectiveness of medical care. Both elements will be analyzed in the following sections.

\section{PCM and patient-oriented research}

One of the fundamental characteristics of medicine in the second half of the $20^{\text {th }}$ century has been its focus on epidemiological and social factors, in an attempt to maximize the health results in the general population. This population-oriented focus is intimately linked with the development of clinical epidemiology and evidencebased medicine (EBM) [24]. This approach has formed the paradigm of scientific medical research, placing RCTs as the gold standard for evaluation of healthcare interventions, contributing to the standardization of healthcare and the development of evidence-based guidelines.

The development of PCM necessitates closing the gap between clinical research based on population studies and clinical practice, with a shift to human-centric factors and ultimately to improve the health outcomes for individual patients [25]. An important objective of patient-oriented research should not be to predict what percentage of patients will respond to a particular intervention, but rather to identify which patients are likely to respond. Table 2 compares the main features of population-oriented research, typical of EBM, and patient-oriented research.

The development of PCM has coincided with that of comparative effectiveness research (CER) [26]. The joint development of PCM and CER is not accidental. CER has been defined as "the generation and synthesis of evidence that compares the benefits and harms of alternative methods to prevent, diagnose, treat, and monitor a clinical condition or to improve the delivery of care. The purpose of CER is to assist consumers, clinicians, purchasers, and policy makers to make informed decisions that will improve healthcare at both the individual and the population levels" [27].

CER and PCM share a common objective: to identify which options are more effective for which patients [28]. For this reason, CER could become an excellent platform from which to develop a patient-oriented research strategy that could serve as a basis for PCM. To achieve this, it will be necessary to change the beliefs that are strongly entrenched in the scientific community. Predictably, in a research environment that is strongly influenced by EBM, large pragmatic RCTs are considered the "logical" starting point for CER [29]. These studies compare clinically relevant options, enroll a diverse clinical population recruited from a variety of practice settings, and measure a broad range of relevant health outcomes [30]. Observational studies using patient records and other databases have also been promoted, particularly in settings where RCTs are not feasible [31].

The objective of these large pragmatic studies is to reach conclusions that are generalizable to all types of patients with a specific disease. From the context of traditional 
Table 2 Characteristics of population- and patient-oriented research

\begin{tabular}{ll}
\hline Population-oriented research & Patient-oriented research \\
\hline Paradigm: Randomized clinical trials & No paradigm: observational and experimental research \\
\hline Focuses on the "generalization" of results & Focuses on the "individualization" of results \\
\hline Efficacy in average patients & Effectiveness in subgroups of patients and individual patients \\
\hline Absolute efficacy & Comparative effectiveness \\
\hline Identify the percentage of patients who will respond to an intervention & Identify which options are more effective for which patients \\
\hline Evaluation of interventions & Evaluation of patients and their diseases \\
\hline Analysis of homogeneity & Analysis of heterogeneity \\
\hline A posteriori subgroup analysis & Subgroups identified a priori \\
\hline Aggregation: the study of commonalities & Disaggregation: the study of differences \\
\hline Inductive logic & Hypothetic-deductive logic \\
\hline Exploratory observations and confirmatory trials & Exploratory trials and confirmatory observations \\
\hline Minimizes the value of observations, exceptions, and case series & Assigns greater value to observations, exceptions, and case series \\
\hline Distinction between clinical practice and research & Integration of clinical practice and research \\
\hline From bench-to-bedside & From bedside-to-bench \\
\hline Evidence-based medicine & Medicine-based evidence \\
\hline
\end{tabular}

clinical research, this is a reasonable objective, as one of the main limitations of RCTs is the generalizability of their results [32]. However, from the context of patient-oriented research, the main limitation of RCTs is not their lack of generalizability, rather the lack of individualization. Therefore, increasing the heterogeneity of the studies, by enrolling many different types of patients, is not the best solution as large RCTs and megatrials do not provide an adequate solution to this limitation, but actually makes it worse, meaning the "forest does not let us see the trees" [4].

Patient-oriented research requires an analysis of the heterogeneity of different kinds of patients [33]. The best decisions for the average patient are not necessarily the best decisions for an individual patient [34-37]. There are often subgroups of patients with different basal risk levels who respond better than the average patient to the same treatment, while other patients are not helped or may even be harmed by this treatment [38]. This heterogeneity of response may be "quantitative", where one of the interventions is superior to another in all the subgroups or "qualitative" when one of the interventions is superior in some subgroups and worse in others [39]. In both of these situations, the strategy of expanding the inclusion criteria in RCTs and evaluating the effects of interventions in heterogeneous groups of patients actually dilute the observed effects, requiring larger sample sizes to detect statistically significant differences.

Some authors have reported that the future of clinical research should lie in the implementation of large RCTs designed to show small differences among heterogeneous groups of patients $[40,41]$. However, are large sample sizes necessary because the differences in efficacy of the treatments being compared are small, or are the differences small because the samples are too large and heterogeneous? If this is the case, perhaps we should consider smaller studies of homogenous subgroups defined $a$ priori to detect clinically important differences.

Subgroup analysis involves improvements to patient classification systems and the development of phenotypic and genotypic markers. Additionally, it will be important to encourage studies focusing on the physiopathological mechanisms of diseases, factors that may determine differences in prognosis and response between subtypes of patients. It is equally important to fully evaluate the biological differences between patients and understand the psychological, social, and cultural differences that could modify personal preferences, as these could influence the patient's preference for a specific alternative treatment, and condition the patient's prognosis and final health outcomes.

A priori subgroup analysis represents an advance over analyses of the average patient, but one of its limitations is that classification is carried out on a single variable, such as sex, age, or the presence of a particular clinical characteristic. Unfortunately, these single variable analyses do not include all relevant patient information. For this reason, it is desirable to develop methods that would provide more complete information on all of the relevant characteristics of all patients, which would facilitate the jump from subgroup analyses to individual analyses. Several interesting methods, based on risk models, have been proposed to predict the effects of a specific treatment in individual patients [42].

The development of patient-oriented research requires a change in the type of reasoning. If the path from an individual patient towards the average patient requires data aggregation, the path back to the individual should follow the inverse process: disaggregation of data, the 
analysis of subgroups and individuals [43]. Although many patients are similar, all patients are different. Similarities between patients explain why inductive reasoning, under which generalizations are drawn from an accumulation of cases, has taken such a strong hold on research. A patient-oriented research perspective demands a shift towards hypothetic-deductive logic, in which RCTs primarily serve to generate a hypothesis (i.e., that one intervention is superior to another in some groups of patients), while observations would test the validity of the hypothesis for individual patients. Consequently, RCTs would be considered as "exploratory" and observations as "confirmatory" [43]. This change in thinking supposes that regulatory approval should no longer be regarded as the final step in hypothesis-testing, but as the hypothesisgenerating step. Currently, most clinical studies are concentrated in the preauthorization phase and are oriented towards hypothesis generation. Predictably, in the future, a greater emphasis will be given to confirmatory investigation, carried out after regulatory approval.

Consistent with this approach, patient-oriented research should give much greater weight to clinical observations. It is important to note the role played by careful observation in the history of clinical studies of new drugs, such as that described by Roland Kuhn on the discovery of the antidepressant imipramine: "I have never used 'controlled double blind studies' with 'placebos', 'standardized rating scales' or the statistical treatment of records of large numbers of patients. Instead I examined each patient individually every day, often on several occasions, and questioned him or her again and again. Many of the patients were also under the observation of my assistants and nursing staff, and I always regarded their proposals and criticisms seriously" [44].

Case reports and case series may provide the weakest level of evidence, but are often the "first line of evidence" $[45,46]$. Individual cases are most useful when they show us the unexpected and, in research, the unexpected can signal a new truth [47]. But, if isolated observations are important, then so is the systematization of observations. Some authors have proposed prospective "formal case studies" to collect pure cases in which to test a priori hypotheses [48]. In a prospective case study, the investigator consciously and explicitly reflects on the theory, and uses it to develop a specific hypothesis or model that is subsequently tested in patients deliberately chosen to either confirm or reject the hypothesis [49].

\section{How can we improve the effectiveness of interventions in clinical practice?}

One of the primary objectives of PCM is that the patients should derive greater benefit from the results of clinical research. In recent years, the concept of translational research has received significant attention.
Translational research is defined as "the effective translation of new knowledge, mechanisms, and techniques generated by advances in basic science research into new approaches for the prevention, diagnosis, and treatment of disease" [50]. The expression "from bench-tobedside" accurately reflects the objective of translational research, a type of research frequently characterized by the lack of communication between basic research and clinical research. This concept has also led to a true "death valley" [51], where basic research findings are rarely transformed into solutions for patients.

One way of increasing the effectiveness of biomedical research is to change the direction of research, in which we start with identifying the true requirements of the patients, the medical problems that still have no answers, or the exceptions that surprise clinicians. It is these questions arising in the clinics that should start the path towards basic research, in a direction "from bedside-to-bench".

The term "translational research" also refers to "translation of the results from clinical studies into everyday clinical practice and health decision-making" [50]. Closing the gap between efficacy and effectiveness is fundamental to ensuring that the results of RCTs are translated into tangible improvements in patient outcomes. It has been claimed that patients could benefit more, and more patients could benefit, if the health services produce better results when delivering existing treatments than by introducing new treatments [52].

One of the reasons that the advances generated by EBM are not put into practice, or are done with considerable delays, is the huge gap between research and clinical practice [53]. Closing the gap between experiments and real life means eliminating the barriers between research and clinical practice. Clinicians should become more engaged in research and be provided with better methodological training. Perhaps more importantly, however, the research should be moved closer to clinics, become simpler, and be better incorporated into the daily activities of clinicians.

It is essential to eliminate the differences between doctors who carry out research and those who do not, between patients who participate in clinical trials and those who do not, and between the case report form of RCTs and the medical record [4]. Every medical act has the structure of an experiment, and every experiment starts with a patient [54]. The results of RCTs can take decades to transfer from the pages of a peer-reviewed medical journal to routine clinical practice, but anecdotes of adverse findings can transform clinical practice in an instant [55].

New information-based technologies, such as electronic medical records (EMR), can help to unite clinical research and medical care [56]. Researchers can exploit EMRs 
containing a vast amount of information generated by each medical interaction. The idea of applying randomization to EMRs would enable "randomized database studies", which combine the advantages of observational studies and experimental studies [57]. The incorporation of decision aids could encourage quicker application of the knowledge acquired during research [58].

To date, EMRs have been used to aggregate data and to carry out large analytical observational studies of the average effectiveness of health interventions. From a patient-centered perspective, EMRs are potentially most useful to disaggregate data and to identify possible differences among patients. EMRs can be used to (1) assess how and in which particular patients interventions are applied in clinical practice; (2) analyze different response patterns, identify patient subgroups, and classify them in accordance with their risk factors and comorbidities; (3) help systematize the exceptions to treatment and the factors responsible for these exceptions; (4) understand a given intervention's risk-benefit ratio at the individual level; (5) record patient preference and perception; and (6) support individual decisions by providing information about each patient. Integration of prediction models, risk calculators, and decision aids in EMRs may facilitate decision-making at the individual level, taking into account the benefits and risks anticipated for each patient [42,59]. Predicting the effects of a treatment for individual patients may enable doctors to practice individualized medicine in an evidence-based manner [42], while considering that the best test for a clinical prediction rule is not accuracy but rather improved clinical outcomes [60].

Closing the gap between efficacy and effectiveness also requires us to carry out strategies and programs that will help make potentially effective treatments truly effective [61]. It it of little use to develop more effective treatments if the patients do not use them correctly. We must stop focusing on measuring noncompliance with treatment, and instead endeavor to improve compliance. Patient-support programs have recently been developed, and are defined as "a service for direct patient-carer interaction/engagement designed to help management of medication and/or disease outcomes (e.g., adherence, awareness and education), or to provide healthcare professionals with support to their patients" [62]. Examples of patient-support programs include (1) compliance programs in which consenting patients given a specific medication are contacted to gauge how well they are managing their medication; (2) call centers where patients or caregivers can contact the company to obtain further information on a treatment or a particular disease area; and (3) nurse educator programs, for which the company has hired third-party nurses to directly interact with patients to help them properly administer their medications and/or manage their disease [62].
Patient-support programs should affect all healthcare professionals that are directly or indirectly related to patient care, including doctors, pharmacists, nurses, and caregivers, as well as the patients themselves. PCM should entail systematic use of such programs, recognizing that optimal treatment does not end with writing the correct prescription, but in the implementation of solutions that improve our knowledge of the disease, enable greater patient involvement in realizing therapeutic benefits, and ultimately achieve better outcomes.

\section{The cost of patient-centered medicine}

Cost control and sustainability of healthcare systems are common goals of the governments of all countries. Prioritization of health interventions taking into account their efficiency, and the use of thresholds of costeffectiveness are controversial topics [63]. For example, assessing the cost-effectiveness of interventions being compared is not within the objectives of the recently established Patient-Centered Outcomes Research Institute [64]. However, if the objective of value-based healthcare systems is to achieve the best health outcomes for patients, within the economic resources available [8], it seems logical that an objective of PCM must be to generate the best possible results for the patients, taking into account what the payer can afford. It is also important to understand that, in public health systems, the payer will be the system itself whereas the patients or insurers are the payers in private health systems.

It has been argued that applying cost-effectiveness criteria implies a loss of clinical freedom [65]. However, the doctor plays a fundamental role in the efficient use of resources, and economic aspects are always present, either implicitly or explicitly, in their decisions. If resources are limited, particularly in the context of public health systems based on the principal of equity, it is not ethical to make clinical decisions without taking into account the associated costs. Therefore, the solution lies in choosing the best options that the health system can offer.

The efficiency of a medical intervention is not an intrinsic characteristic, but rather depends on how it is being used in clinical practice, including in what subgroup of patients, at what dose, and for how long. An essential responsibility of the doctor is to individualize these interventions in terms of cost-effectiveness, while maximizing the outcomes according to the clinical characteristics and preferences of the patients [66]. The choice of an intervention according to its efficiency should not be considered the end, but just the start of clinical freedom [67].

\section{Summary}

The growing role played by patients within the healthcare system, which could be summarized with the slogan 
"nothing about me without me"; the development of personalized therapies, where "one size does not fit all"; the problems associated with EBM, focusing on the evaluation of medical interventions in average patients; and the difficulties in patients obtaining some tangible benefit from the results of clinical research, are just some of the factors that have propelled the recent and rapid development of PCM. The objective of PCM is to improve the health outcomes for individual patients, within the context of everyday clinical practice, taking into account the objectives, preferences, and values of each patient, as well as the available economic resources.

PCM requires a paradigm change in the way medicine is being practiced. We are now moving from a providercentered model based on the beneficence and authoritarianism of the doctor, to a patient-centered model, based on a principle of autonomy. The patient's preferences, objectives and values should play a fundamental role in shared decision-making, and the doctor becomes the "enabler who supports the patient to make informed decisions". The final objective should not be to know what is the best treatment for the average patient, but to improve the health outcomes of individual patients, while ensuring the results of medical research have a real impact on healthcare.

This change in paradigm requires a change in the relationship between doctors and patients, in the ethical principles that control the relationship between them, and in education and medical legislation. It also has important implications for research processes. The development of patient-oriented-research is essential for PCM. It seems logical that the methods underpinning EBM and PCM speak different languages. If EBM is characterized by speaking the language of populations, it should concentrate on the evaluation of health interventions, analysis of aggregated data, variables important to doctors and health regulators, inductive reasoning, the predominance of quantitative methods, the analysis of large sample sizes, and the study of similarities and homogeneity; the methods of PCM should speak the language of individuals by focusing on the evaluation of patients, disaggregation of data, variables important to patients, hypothetical/deductive reasoning, the use of qualitative methodology, analysis of subgroups and individuals, and the analysis of heterogeneity and exceptions [68].

Further developments in information-based technologies can help to close the existing gap between research and clinical practice, without which it would be very difficult to evaluate the effectiveness of healthcare interventions or translate the results of clinical research into tangible benefits for patients. Bringing research and clinical practice closer together will provide advances in the road towards EBM [69], lending greater weight to observations and exceptions, and ultimately ensure that the directionality of translational research is "from bedsideto-bench".

Finally, it must be emphasized that PCM is not incompatible with EBM. A collision between the two must be avoided. Both approaches converge on healthcare from different points of view, and must be considered complementary. If EBM follows the path from the individual patient to the average patient, PCM follows the opposite path, from the average patient to the individual patient. It is not possible to practice PCM that is not based on evidence, nor is it possible to practice EBM at a distance from the individual patient.

Competing interests

JAS is employed by Eli Lilly \& Company.

\section{Acknowledgements}

The author thanks to Edanz Group Global Ltd for their editing support on this manuscript.

Received: 2 October 2012 Accepted: 31 December 2012

Published: 8 January 2013

\section{References}

1. Truog RD: Patients and doctors. The evolution of a relationship. $N$ Engl J Med 2012, 366:581-585.

2. Laine C, Davidoff F: Patient-centered medicine. A professional evolution. JAMA 1996, 257:152-156

3. Meyer UA: Personalized medicine: a personal view. Clin Pharmacol Ther 2012, 91:373-375.

4. Sacristan JA: Patient-centered medicine and comparative effectiveness research. Med Clin (Barc) 2011, 136:438-440.

5. Godlee F: Outcomes that matter to patients. BMJ 2012, 344:e318.

6. Gandhi GY, Murad MH, Fujiyoshi A, Mullan RJ, Flynn DN, Elamin MB, Swiglo BA, Isley WL, Guyatt GH, Montori VM: Patient-important outcomes in registered diabetes trials. JAMA 2008, 299:2543-2549.

7. Rahimi K, Malhotra A, Banning A, Jenkinson C: Outcome selection and role of patient reported outcomes in contemporary cardiovascular trials: systematic review. BMJ 2010, 341:c5707.

8. Porter M: A strategy for health care reform. Towards a value-based system. N Engl J Med 2009, 361:109-112.

9. Reuben DB, Tinetti ME: Goal-oriented patient care. An alternative health outcomes paradigm. N Engl J Med 2012, 366:777-779.

10. Jonas WB: Scientific evidence and medical practice. Arch Intern Med 2009, 169:649-650.

11. US Food and Drug Administration: Guidance for industry patient-reported outcome measures: use in medical product development to support labeling claims; 2009. http://purl.access.gpo.gov/GPO/LPS113413.

12. Coons SJ, Kothari S, Burke LB: The patient-reported outcome (PRO) consortium: filling measurement gaps for PRO end points to support labeling claims. Clin Pharmacol Ther 2011, 90:743-748.

13. Vieta A, Badia X, Sacristan JA: A systematic review of patient-reported and economic outcomes: value to stake holders in the decision-making process in patients with type 2 diabetes mellitus. Clin Ther 2011, 33:1225-1245.

14. Vieta A, Badia X, Álvarez E, Sacristan JA: Which nontraditional outcomes should be measured in healthcare decision-making in schizophrenia? A systematic review. Perspect Psichiatry Care 2012, 48:198-207.

15. Lesko $L$, Zineh I, Huang S-M: What is clinical utility and why should we care. Clin Pharmacol Ther 2010, 88:729-733.

16. Rothrock NE, Kaiser KA, Cella D: Developing a valid patient-reported outcome measure. Clin Pharmacol Ther 2011, 90:737-742.

17. Fischer D, Stewart AL, Bloch DA, Lorig K, Laurent D, Holman H: Capturing the patient's view of change as a clinical outcome measure. JAMA 1999 , 282:1157-1162.

18. Krahn M, Naglie G: The next step in guideline development. Incorporating patient preferences. JAMA 2008, 300:436-438.

19. Barry MJ, Edgman-Levitan S: Shared decision making. The pinnacle of patient-centered care. N Engl J Med 2012, 366:780-781. 
20. Marshall M, Bibby J: Supporting patients to make the best decisions. $B M J$ 2011, 342:775-777.

21. Keirns CC, Goold SD: Patient-centered care and preference-sensitive decision making. JAMA 2009, 302:1805-1806.

22. Rodríguez-Artalejo F, Ortún V: General treatments versus personalized treatments. Gest Clin Sanit 2003, 5:87-88.

23. Stacey D, Bennett CL, Barry MJ, Col NF, Eden KB, Holmes-Rovner M, Llewellyn-Thomas H, Lyddiatt A, Légaré F, Thomson R: Decision aids for people facing health treatment or screening decisions. Cochrane Database Syst Rev 2011, 10. CD001431.

24. Sackett DL, Richardson WS, Rosenberg W, Haynes RB: Evidence-based medicine: how to practice and teach EBM. New York: Churchill Livingstone; 1997.

25. Hartzband P, Groopman J: Keeping the patient in the equation. Humanism and health care reform. N Engl J Med 2009, 361:554-555.

26. Institute of Medicine: Initial National Priorities for Comparative Effectiveness Research. Washington, DC: The National Academies Press; 2008.

27. Sox HC, Greenfield S: Comparative effectiveness research: a report from the Institute of Medicine. Ann Intern Med 2009, 151:203-205.

28. DeMaría AN: Comparative effectiveness research. J Am Coll Cardiol 2009, 53:973-975

29. Luce BR, Kramer JM, Goodman SN, Connor JT, Tunis S, Whicher D, Schwartz $S$ : Rethinking randomized clinical trials for comparative effectiveness research: the need for transformational change. Ann Intern Med 2009, 151:206-209.

30. Tunis SR, Stryer DB, Clancy CM: Practical clinical trials: increasing the value of clinical research for decision making in clinical and health policy. JAMA 2003, 290:1624-1632.

31. Concato J, Lawler EV, Lew RA, Gaziano M, Aslam M, Huang GD: Observational methods in comparative effectiveness research. Am J Med 2010, 123:e16-e23.

32. Rawlins M: Harveian Oration. De testimonio: on the evidence for decisions about the use of therapeutic interventions. Lancet 2008 372:2152-2161.

33. Kravitz RL, Duan NH, Braslow J: Evidence-based medicine, heterogeneity of treatment effects, and the trouble with averages. Milbank Q 2004 82:661-687.

34. Rothwell PM: Can overall results of clinical trials be applied to all patients? Lancet 1995, 345:1616-1619.

35. Mant M: Can randomized trials inform clinical decisions about individual patients? Lancet 1999, 353:743-6.

36. Simon G: Choosing a first line antidepressant: equal on average does not mean equal for everyone. JAMA 2001, 286:3003-3004.

37. Horwitz Rl, Singer BH, Makuch RW, Viscoli CM: Can treatment that is helpful on average be harmful to some patients? A study of the conflicting information needs of clinical inquiry and drug regulation. J Clin Epidemiol 1996, 49:395-400

38. Ioannidis JP, Lau J: Uncontrolled pearls, controlled evidence, metaanalysis and the individual patient. J Clin Epidemiol 1998, 51:709-711.

39. Wang $R$, Lagakos SW, Ware JH, Hunter DJ, Drazen JM: Statistics in medicine. Reporting of subgroup analyses in clinical trials. N Engl J Med 2007, 357:2189-2194.

40. Yusuf $S$, Collins R, Peto R: Why do we need some large simple randomized trials. Stat Med 1984, 3:409-422.

41. Peto R, Baigent C: Trials: the next 50 years. Large scale randomized evidence of moderate benefits. BMJ 1998, 317:1170-1171.

42. Dorresteijn JAN, Visseren FL, Braunwald PMRE, Wassink AMJ, Paynter NP, Steyerberg EW, van der Graaf Y, Cook NR: Estimating treatment effects for individual patients based on the results of randomized clinical trials. BMJ 2011, 343:d5888

43. Sacristán JA: Exploratory trials, confirmatory observations: a new reasoning model in the era of patient-centered medicine. BMC Med Res Methodol 2011, 11:57.

44. Healy D: The Psychopharmacologists, Volume II. London: Altman; 1998.

45. Jenicek M: Clinical case reporting in evidence-based medicine. Oxford: Butterworth-Heinemann; 1999:117.

46. Vandenbroucke JP: In defense of case reports and case series. Ann Intern Med 2001, 134:330-334.

47. Aronson JK, Hauben M: Anecdotes that provide definitive evidence. BMJ 2006, 333:1267-1269.

48. Charlton BC: Individual case studies in primary health care. Fam Pract 1999, 16:1-2.
49. Charlton BG: Individual case studies in clinical research. J Eval Clin Practice 1998, 4:147-155.

50. Fontanarosa $\mathrm{PB}$, DeAngelis CD: Basic science and translational research in JAMA. JAMA 2002, 287:1728.

51. Butler D: Translational research: crossing the valley of death. Nature 2008, 453:840-842.

52. Woolf $\mathrm{SH}$ : The meaning of translational research and why it matters. JAMA 2008, 299:211-213

53. Eichler HG, Abadie E, Breckenridge A, Flamion B, Gustafsson LL, Leufkens $H$, Rowland M, Schneider CK, Bloechl-Daum B: Bridging the efficacyeffectiveness gap: a regulator's perspective on addressing variability of drug response. Nature Rev Drug Discov 2001, 10:495-506.

54. Feinstein AR: Clinical judgment revisited: the distraction of quantitative models. Ann Intern Med 1994, 120:799-805.

55. Stuebe AM: Level IV evidence. Adverse anecdote and clinical practice. N Engl J Med 2011, 365:8-9.

56. Wang CJ, Huang AT: Integrating technology into health care. What will it take? JAMA 2012, 307:569-570

57. Sacristan JA, Soto J, Galende I, Hylan TR: Randomized database studies: a new method to assess drugs' effectiveness? J Clin Epidemiol 1998, 51:713-715.

58. Fraenkel L, Fried TR: Individualized medical decision making. Necessary, achievable, but not yet attainable. Arch Intern Med 2010, 170:566-569.

59. Wilke RA, Xu H, Denny JC, Roden DM, Krauss RM, Krauss RM, McCarty CA, Davis RL, Skaar T, Lamba J, Savova G: The emerging role of electronic medical records in pharmacogenomics. Clin Pharmacol Ther 2011, 89:379-386.

60. Grady D, Berkowitz SE: Why is a good clinical prediction rule so hard to find? Arch Intern Med 2011, 171:1701-1702.

61. Bero LA, Grilli R, Grimshaw JM, Harvey E, Oxman AD, Thomson MA: Closing the gap between research and practice: an overview of systematic reviews of interventions to promote the implementation of research findings. BMJ 1998, 317:465-468.

62. The Association of the British Pharmaceutical Industry: Guidance notes for patient safety and pharmacovigilance in patient support programmes; 2011. http://www.abpi.org.uk/our-work/library/guidelines/Pages/patient-safetypharmacovigilance-aspx.

63. Neumann PJ: What we talk about when we talk about health care costs. N Engl J Med 2012, 366:5885-5886.

64. Brooks RH: Can the patient-centered outcomes research institute become relevant to controlling medical costs and improving value? JAMA 2011, 306:2020-2021.

65. Hampton JR: The end of clinical freedom. Br Med J (Clin Res Ed) 1983, 287:1237-1238.

66. Huang ES, Shook M, Jin L, Chin MH, Meltzer DO: The impact of patient preferences on the cost-effectiveness of intensive glucose control in older patients with new-onset diabetes. Diabetes Care 2006, 29:259-264.

67. Sacristán JA, Costi M, Valladares A, Dilla T: Health economics: the start of clinical freedom. BMC Health Serv Res 2010, 10:183.

68. Sacristán JA: Evidence from randomized controlled trials, meta-analyses, and subgroup analyses. JAMA 2010, 303:1253-1254.

69. Sacristán JA: Medicine-based evidence. Med Clin 1999, 112(Suppl 1):9-11.

\section{doi:10.1186/1472-6947-13-6}

Cite this article as: Sacristán JA: Patient-centered medicine and patientoriented research: improving health outcomes for individual patients. BMC Medical Informatics and Decision Making 2013 13:6.

\section{Submit your next manuscript to BioMed Central and take full advantage of:}

- Convenient online submission

- Thorough peer review

- No space constraints or color figure charges

- Immediate publication on acceptance

- Inclusion in PubMed, CAS, Scopus and Google Scholar

- Research which is freely available for redistribution 